\title{
MORTANDAD EN LA FRONTERA
}

\author{
ANDREAS FELDMANN \\ JORGE DURAND
}

RESUMEN: En el presente trabajo se examina el fenómeno de la muerte de migrantes en el corredor migratorio de América del Norte, en particular en la frontera entre México y Estados Unidos, desde la perspectiva de los derechos humanos. Nuestro principal argumento es que la actual política migratoria estadounidense busca de manera explícita disuadir a los migrantes de cruzar la frontera al aumentar los costos y los riesgos del cruce irregular. Al desviar los flujos migratorios hacia áreas más agrestes y desoladas donde el cruce de la frontera encierra evidentes riesgos, las autoridades estadounidenses están sometiendo a los migrantes a una especie de darwinismo social, en el que sólo los más fuertes y con mayores recursos logran sortear los peligros del cruce y llegar a su destino. $\mathrm{Al}$ mismo tiempo, sostenemos que tanto en términos del derecho internacional como de los derechos humanos existe un vacío legal que impide promover medidas concretas para proteger la vida de los migrantes irregulares que intentan ingresar a Estados Unidos por lugares no habilitados. 


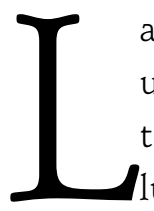

a migración internacional siempre se ha considerado como una aventura, un paso a lo desconocido, el tránsito a un mundo diferente lleno de misterio y oportunidades o, por lo menos, con más oportunidades que el ugar de origen. Sin embargo, hoy en día se ha convertido en una aventura peligrosa, costosa y arriesgada. No hay cálculos oficiales sobre el número de muertos en la aventura migratoria contemporánea, pero fácilmente rebasan varios miles por año.

Por lo general son los estrechos los que cobran mayor número de víctimas. El mar en esos lugares suele ser traicionero y tener corrientes muy peligrosas. El estrecho de Florida entre Cuba y Miami ha cobrado innumerables vidas de «balseros» en su intento por llegar a Estados Unidos; en el estrecho de la Mona, entre República Dominicana y Puerto Rico, el mar se ha engullido innumerables "yolas», pequeñas embarcaciones que utilizan los pescadores de la zona; en el estrecho de Gibraltar muchas "pateras» que zarpan del Magreb no han podido llegar a España, la puerta de entrada a Europa. No se puede dejar de nombrar a los «cayucos» africanos que parten de Mauritania y Senegal y tratan de llegar a las islas Canarias, que son territorio Europeo. En todos estos casos entre las víctimas se mezclan migrantes económicos, refugiados y peticionarios de asilo. Las muertes en alta mar son muchas veces desconocidas y escapan a cualquier contabilidad. Lo que sí se puede afirmar es que son muy numerosas.

El mar ha sido un espacio transitado por migrantes de todas las épocas. Pero en los años setenta entraron en el escenario mundial los «boat people», emigrantes y solicitantes de asilo. El nombre parece haber surgido, cuando en esta década se encontraron en alta mar a cientos de vietnamitas que huían en frágiles embarcaciones. Luego en los ochenta volvieron a ser noticia los «boat people» que salían de Haití hacia Florida. En el 2001 una embarcación indonesa con cientos de migrantes que se dirigían a Australia, fue rescatada. En las costas del Pacífico también se han rescatado embarcaciones pesqueras atiborradas de migrantes provenientes de Perú y Ecuador, que intentan llegar a México para luego cruzar a Estados Unidos.

Por su parte, en el corredor norteamericano que se inicia en Centro América atraviesa México y se dirige hacia Estados Unidos, también se ha registrado un creciente número de muertes de migrantes. México es la puerta de entrada hacia Estados Unidos, puerta trasera, pero muy amplia y porosa; de ahí que migrantes de todo el mundo, muy especialmente latinoamericanos, se dirijan a este país con la intención de cruzar hacia Estados Unidos.

Existe un incentivo adicional, además de la vecindad geográfica, los mexicanos han desarrollado un amplio entramado de servicios de «coyotaje» para cruzar la frontera de manera subrepticia. El fenómeno no es nuevo, desde fines del siglo XIX existían los coyotes, que ingresaban ilegalmente ciudadanos chinos y posteriormente japoneses, impedidos de entrar a Estados Unidos por las leyes de exclusión racial (Ryo 2006). 
Como quiera, siempre ha habido riesgos al cruzar la frontera entre México y Estados Unidos, especialmente el río Bravo, que hace honor a su nombre. Sin embargo, diversas fuentes coinciden en señalar que se ha dado un incremento notable (más del 100\%) en el número de víctimas en los últimos 15 años, especialmente a partir de operativos de control fronterizo como Bloqueo y Guardián, que empezaron en 1993 y 1994.

El tema ha sido analizado a distintos niveles, tanto por académicos y universitarios como por organismos oficiales, abogados, fundaciones y organizaciones de apoyo a migrantes. Un trabajo seminal fue el de Karl Eschbach y colegas, del Center for Immigration Studies de la Universidad de Houston, que empezaron a publicar sobre el tema en 2001 y 2003. Han continuado la tarea en el campo académico Cornelius (2001), Belinda Reyes (2002), Scharf (2006) y Sapkota et al. (2006), Marroni y Meneses (2006). Por otra parte, el tema se analiza en documentos oficiales como la querella presentada en 1999 ante la Comisión Interamericana de Derechos Humanos (CIDH 2005) por las muertes causadas por la Operación Gatekeeper y el informe al Senado elaborado por la United States Goverment Accountability Office (GAO 2006) que aporta información, evaluación y análisis recientes sobre el cruce fronterizo.

En el presente trabajo nos interesa replantear el tema de la muerte en la frontera en el corredor migratorio de América del Norte, actualizar la información existente, proponer una nueva perspectiva de análisis y discutir la problemática en general desde la perspectiva de los derechos humanos. Nuestro principal argumento es que la actual política migratoria estadounidense busca de manera explícita disuadir a los migrantes de cruzar la frontera al aumentar los costos y los riesgos del cruce irregular. Al desviar los flujos migratorios hacia áreas más agrestes y desoladas donde el cruce de la frontera encierra evidentes riesgos, las autoridades estadounidenses están sometiendo a los migrantes a una especie de darwinismo social en el que sólo los más fuertes y con mayores recursos logran sortear los peligros del cruce y llegar a su destino. Por otro lado, sostenemos que tanto en términos del derecho internacional como de los derechos humanos existe un vacío legal que impide promover la protección de la población migrante

En la primera parte de este trabajo describimos el fenómeno de las muertes en la frontera a partir de información estadística, donde se pone en evidencia que las medidas puestas en práctica no han provocado cambios significativos en los flujos migratorios pero si ha redundado en incrementar el nivel de exposición de los migrantes. En la segunda sección, examinamos el problema de la muerte de los migrantes desde el punto de vista del derecho internacional de los derechos humanos y los vacíos legales que existen. Finalmente, presentamos conclusiones y sugerimos algunas avenidas tentativas para promover un debate profundo que aborde este problema y mitigue la exposición de los migrantes que ponen en riesgo su integridad física y sus vidas. 
A comienzos de la década de los noventa, la prensa, los políticos y los funcionarios encargados de la migración comprobaron lo que se ha llamado «las consecuencias no deseadas» de la reforma migratoria de 1986 (IRCA). Es decir, que la reforma no había cumplido con su principal objetivo que era detener el flujo migratorio de indocumentados.

La reforma migratoria de 1986 fue un parte aguas para el patrón migratorio mexicano, principalmente por las consecuencias que acarreó el proceso de legalización masivo de migrantes y el posterior control fronterizo (Massey, Durand y Malone 2002). No obstante, durante los primeros años de IRCA (1986-1992) todo continuaba más o menos igual. La migración mexicana indocumentada seguía su propio y tradicional curso sin mayores sobresaltos. Lo único novedoso era la presencia, cada vez mayor, de migrantes centroamericanos provenientes de El Salvador, Guatemala, Honduras y Nicaragua, que huían de las guerras y sus consecuencias y que se sumaban y mimetizaban con el flujo migratorio mexicano.

La mayor cantidad del flujo indocumentado se concentraba en el área de Tijuana -San Diego, donde era muy fácil pasar a San Isidro. Cientos de migrantes esperaban el anochecer para luego correr hacia el otro lado donde los esperaba la Patrulla Fronteriza. Era el juego del gato y el ratón, todas las noches eran capturados varios cientos de migrantes y otros tantos lograban cruzar (Durand y Massey 2003). Varios indicadores permiten valorar los pocos cambios que se dieron en el control fronterizo entre 1986 (IRCA) y 1993 (Operación Bloqueo): aumentó muy poco el número de patrulleros y las horas de vigilancia, la probabilidad de aprehensión no varió significativamente. Lo único que cambió fue que se duplicó el presupuesto de la Border Patrol, sobre todo para el uso de nueva tecnología.

\section{CUADRO 1}

Indicadores básicos de control fronterizo entre 1986 (IRCA) y 1993

(Operación Bloqueo)

\begin{tabular}{|c|c|c|c|c|}
\hline AÑO & NÚMERO DE PATRULLEROS & HORAS DE VIGILANCIA & PROBABILIDAD DE APRENSIÓN & PRESUPUESTO DE LA BORDER P \\
\hline 1986 & 3,683 & 2.4 millones & 0.301 & 150 Millones \\
\hline 1993 & 3,965 & 2.7 millones & 0.325 & 354 Millones \\
\hline
\end{tabular}

Mexican Migration Project. http://mmp.opr.princeton.edu/ 2007.

No obstante, en Estados Unidos la prensa y los políticos empezaron a alarmarse por la situación del cruce fronterizo. La migración indocumentada, tradicionalmente opaca, se había tornado visible, especialmente por los reportajes televisados que destacaban lo fácil y barato que resultaba cruzar la frontera, sobre todo en el área de San Diego-Tijuana. Consecuentemente, se hizo evidente que la reforma migratoria de 1986 (IRCA) no servía para el propósito de detener el flujo indocumentado. 
Como quiera, el planteamiento de IRCA estaba bien estructurado. El problema es que no se aplicó en sus cuatro dimensiones: amnistía (LAW), trabajadores especiales (SAW), control fronterizo y control interior. La burocracia fue muy efectiva en cuanto al proceso de legalización, que en total otorgó documentos a 3.2 millones de indocumentados (LAW y SAW), de los cuales 2.3 millones eran mexicanos. Sin embargo, muy poco se hizo en los primeros años en cuanto a control fronterizo y prácticamente nada en cuanto al control interior y las sanciones a los empleadores que contrataran indocumentados (Massey, Durand y Malone: 2002; Durand y Massey 2003).

Después de varias décadas de debate se había podido superar la disposición legal conocida como Texas Proviso (1951), que eximía de sanciones a los empleadores que contrataran indocumentados. Con IRCA cambió la legislación, pero los

\section{GRÁFICA 1}

Aprensiones en diferentes sectores de la frontera, 1993

Sectores más grandes de la patrulla fronteriza

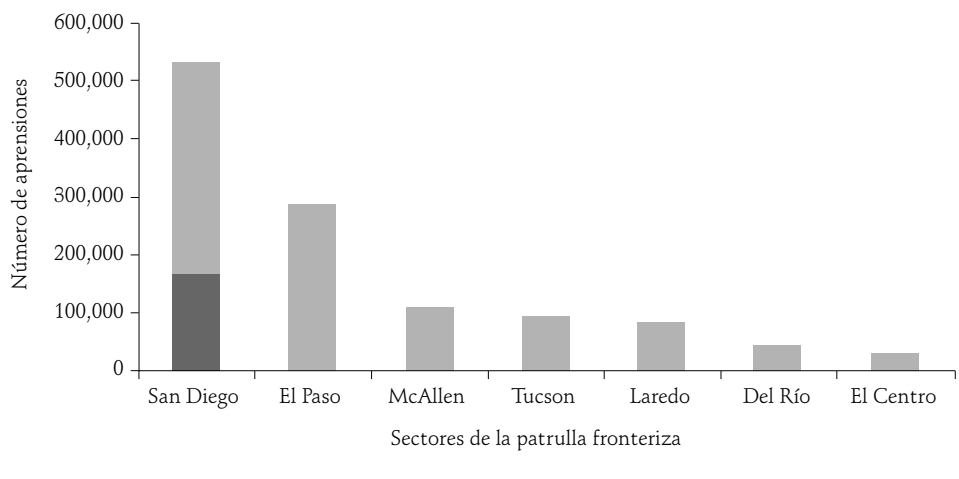

$\square$ Aprensiones en Imperial Beach $\square$ Aprensiones por sector

Fuente: INS, 1995

GRÁFICA 2

Migrantes mexicanos legalizados 1985-2005

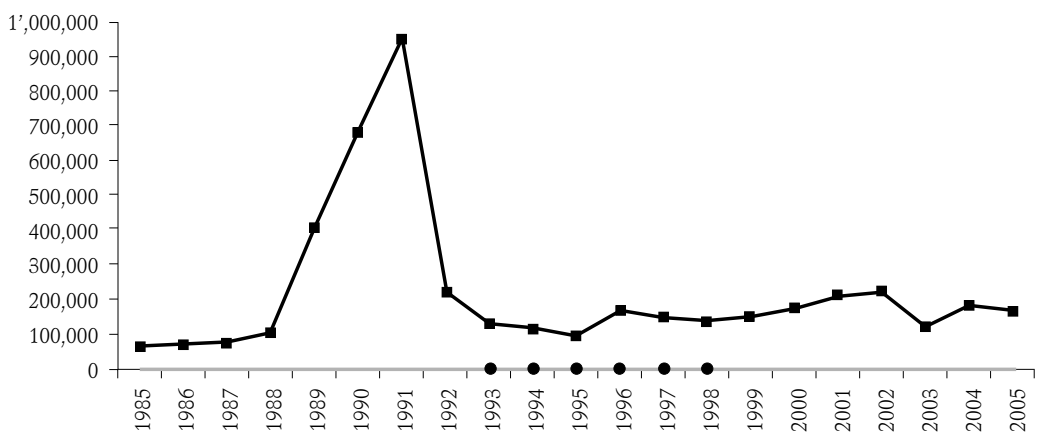

Fuente: elaborado a partir de información oficial sistematizada por el Mexican Migration Project: http://mmp.opr.princeton.edu/ 
empleadores lograron evadir fácilmente la responsabilidad legal de contratar indocumentados, al no poder determinar o validar la autenticidad de los documentos. Existen varios programas pilotos para verificar la autenticidad de los documentos y los SSN (Social Security Number), pero son muy limitados y han dado pocos resultados.

Los trabajadores indocumentados se convirtieron en migrantes "clandestinos» al contar, en la práctica, con documentos falsos que compraban a precios razonables a las mafias de falsificadores. Una vez que cruzaban la frontera podían quedar tranquilos porque prácticamente nadie los molestaba (Massey, Durand y Malone 2003). En 1995 el ins deportó tan sólo a 12,000 indocumentados desde su lugar de trabajo, mientras que en la frontera deportó a 1'340,000 (menos del 1\%) (Mexican Migration Project 2007). La Operación Vanguardia, que pretendía controlar a la población indocumentada en las plantas procesadoras de carne, detectó en 1999 que en 40 plantas habían unos 4,500 casos irregulares, pero finalmente pudo deportar a únicamente 34 trabajadores. Los industriales acusaron al Servicio de Inmigración y Naturalización (INS) de querer llevarlos a la quiebra. ${ }^{1}$

Fue hasta el año de 1993 cuando empezaron a tomarse medidas serias de control fronterizo. En El Paso, Texas, el Superior Regional de la Patrulla Fronteriza, Silvestre Reyes, puso en marcha un programa piloto llamado Operación Bloqueo. Al parecer fue una iniciativa personal que no formaba parte de un programa general. Sin embargo, el éxito repentino de la medida, que consistía en vigilar estrechamente la frontera en la zona urbana, se convirtió posteriormente en política oficial de Estados Unidos (Cortés 2003).

De este modo, en 1994 durante el gobierno del Presidente Clinton, se incrementó el presupuesto y se definió una Estrategia Nacional para la Patrulla Fronteriza, sintetizada en el concepto de prevention through deterrence, prevensión a través de la disuasión (IACHR 2005; GAO 2006). Al parecer se contrató una asesoría militar para redefinir las acciones de control. La recomendación fue cambiar radicalmente de estrategia. Ya no era prioritario detener y deportar indocumentados, sino más bien disuadirlos deterring then rather than trying to aprehend at the border or in the interior (Wayne Cornelius 2001).

En parte, se trataba de seguir el modelo aplicado en El Paso, que consistía en vigilar de manera muy estricta la frontera en la zona urbana. De este modo los migrantes indocumentados dejarían de ser «visibles», al escoger otras rutas mucho más alejadas, difíciles y peligrosas (Cortés 2003) y la prensa, los políticos y determinados sectores de la sociedad podrían dormir tranquilos.

Un año después de la entrada en vigor del operativo en El Paso, en 1994 se puso en marcha la Operación Guardián en el área de San Diego-Tijuana, que era

\footnotetext{
${ }^{1}$ En la actualidad las redadas se han multiplicado y se han hecho más efectivas. En el 2007 fueron capturaros más de 1300 inmigrantes indocumentados en el sur de California. La Opinión, 4 de Octubre de 2007.
} 
la zona con mayor tránsito de migrantes indocumentados (Ver gráfica 1). El resultado fue nuevamente vistoso y exitoso en cuanto al decrecimiento significativo del número de aprensiones. Los migrantes tuvieron que abandonar su punto de reunión, llamado el soccer field y su ruta del Cañón Zapata y se movieron al "Bordo», pegado a la carretera que va a la playa. Luego se construyeron bardas y finalmente se amuralló toda el área vecina de Tijuana, incluyendo parte de la zona marítima. Los migrantes se tuvieron que dirigir hacia otras zonas y allí se incrementó el número de aprensiones.

Las consecuencias, en este caso previstas, nuevamente se hicieron presentes. El desvío del flujo hacia zonas montañosas y desérticas empezó a cobrar su precio en vidas y en dólares. La política disuasiva de incrementar costos y riesgos comenzaba a dar sus frutos (Ver gráfica 3).

\section{GRÁFICA 3}

Costos por servicio de coyote en la frontera mexicana 1980-2004

(Costos normalizados al valor del dolar del 2005)

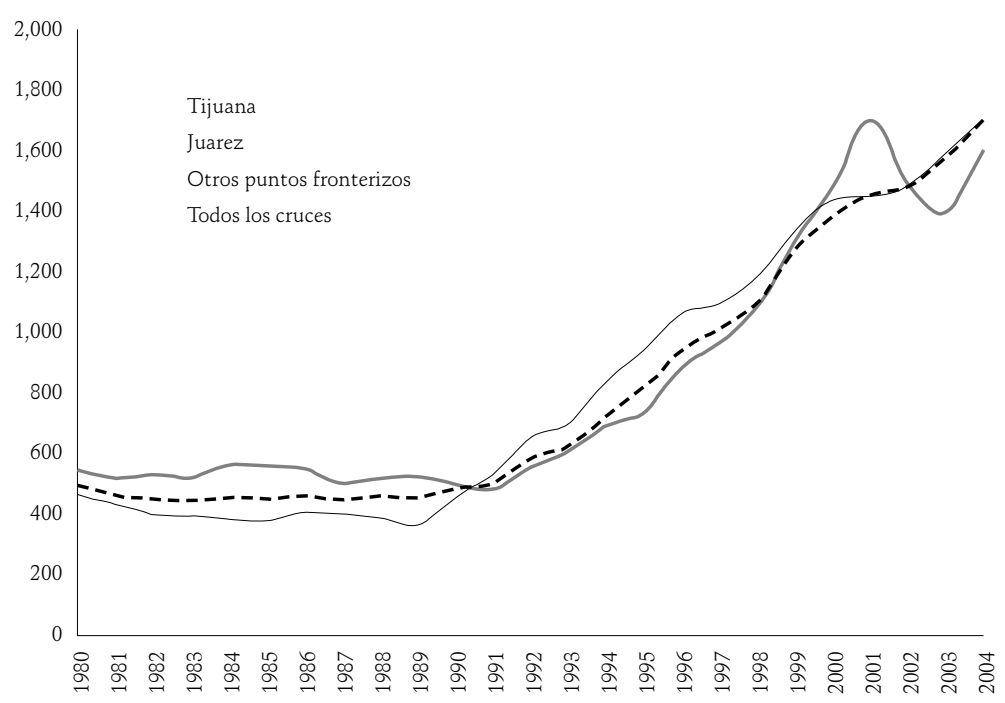

Fuente: Elaborado a partir de datos del Mexican Migration Project: MMP118 http://mmp.opr.princeton.edu/

Los primeros en advertir y denunciar la situación fueron los investigadores del Center for Immigration Resarch de la Universidad de Houston, quienes empezaron a desbrozar el camino, recolectando información bastante dispersa, poco confiable y muchas veces no comparable. Como quiera, una vez definidos los criterios, pudieron establecer que en 1993 se registraron 180 muertes y a partir de los operativos de control fronterizo el número se incremento hasta 370 en el año 2000 (Eschbach et al. 2003). Con fuentes estadísticas más confiables y con registros de ADN, la Secretaría de Relaciones Exteriores de México informó que en el año 2005 se habían registrado 443 muertes de migrantes en ambos lados de la frontera, y que en el año 2006 la cifra fue de 425 (La Jornada, 23 de abril de 2007). 
No entran en esta contabilidad los migrantes que mueren al interior de Estados Unidos, en accidentes de coche o en los contenedores herméticos de los trailers, tampoco se contabilizan a los centroamericanos fallecidos en el camino, durante su tránsito por México (la Ruta de la Muerte) de lo cual existe muy poca información (CIDH 2004).

En relación con lo previsto, las detenciones bajaron significativamente en las áreas urbanas de San Diego y el Paso y se incrementaron notablemente en las áreas de Tucson, Yuma y El Centro. Se había logrado el objetivo principal de desviar los flujos hacia las zonas más peligrosas y allí empezaron a aparecer los cadáveres. Con base en datos de la EMIF, se documenta el cambio que se dio en las deportaciones de migrantes, incluso dentro del mismo estado de Sonora. Primero las deportaciones se concentraron en el área de Nogales (1995-2000) y luego pasaron a Sásabe, en la zona del desierto de Altar (2000-2005).

De acuerdo con los datos del mmp, el paso por el estado de Baja California, colindante con California dejó de ser el principal lugar de cruce de migrantes indocumentados en el año 1996 y el estado de Sonora empezó a cobrar inusitada importancia a partir de 1993. Ambos estados concentran al 70\% de la población indocumentada que cruza por esa zona. Por su parte, los otros estados fronterizos: Chihuahua, Coahuila y Tamaulipas mantienen una tendencia constante, cercana al 10\%. Otras fuentes, confirman estas mismas tendencias (Eschbach et al., 1999, 2001; GAO, 2006).

\section{GRÁFICA 4}

Estados mexicanos por donde cruzan los migrantes indocumentados, MMP, 114 comunidades, 1990 a 2003, base de datos 2007

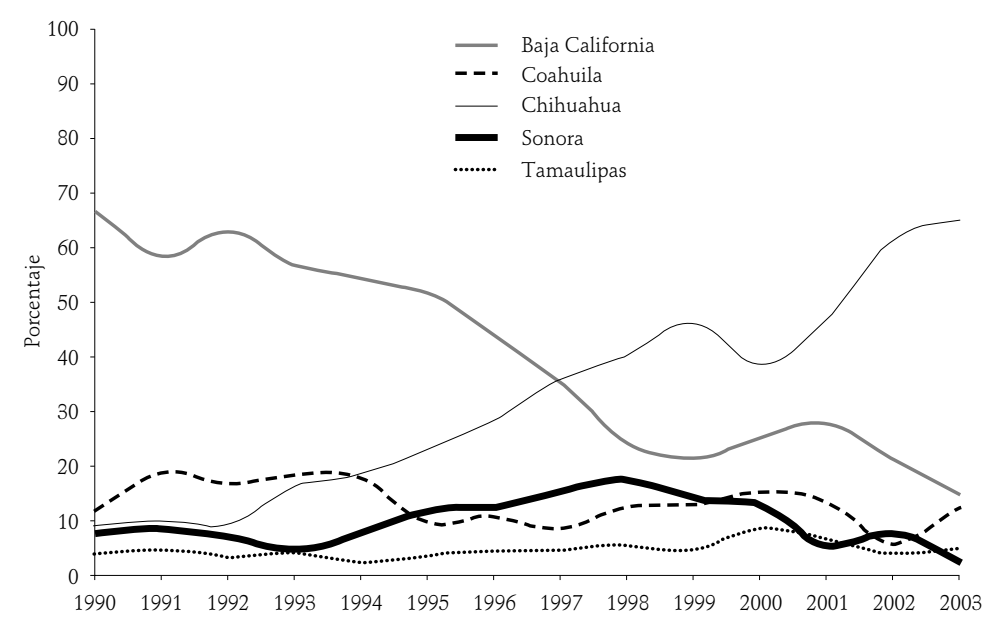

Fuente: Elaborado a partir de datos del Mexican Migration Project, MMP, 114 comunidades, 2007.

A pesar de haberse demostrado con datos confiables el incremento notable en las muertes de los migrantes, como consecuencia de la aplicación de la nueva 
política migratoria, el gobierno de Estados Unidos no se siente responsable y alega que la responsabilidad de las muertes recae en los migrantes que deciden exponerse a cruces por lugares no habilitados. En otras palabras, la naturaleza se encarga de hacer su labor y de ello son responsables los mismos migrantes que asumen el riesgo de ingresar por zonas peligrosas. Se da una especie de selección natural donde los más fuertes, mejor entrenados, más aptos y con mayores recursos pueden sobrevivir a la prueba. En varias ocasiones los migrantes mismos reconocen que tienen que dejar abandonados a sus compañeros que tuvieron algún accidente, que no podían seguir caminando o que retrazaban a todo el grupo. En muchos de estos casos son las mujeres y los niños los que están más expuestos a perder la vida (Marroni y Meneses 2006).

Las estadísticas confirman que es la naturaleza, the natural landscape la principal causa de muerte de los migrantes. La deshidratación y la hipotermia se encuentran en primer lugar, seguidas por los accidentes naturales y mordeduras de animales venenosos. Según las estadísticas de GAO (2006) la causa principal de muerte en la frontera se atribuye a causas relacionadas con accidentes de tráfico (35\%) y en segundo lugar a la «exposición» a altas o bajas temperaturas (23\%). Llama la atención que las estadísticas oficiales del GAO (2006) destaquen que se han reducido notablemente las muertes violentas. ${ }^{2}$ Los datos del GAO (2006) indican que la proporción de muertes por asesinato (14\%) es ligeramente inferior al de muertes por «intervención legal» (15\%); es decir, muertes causadas directamente por la Patrulla Fronteriza. A pesar de que existe un convenio entre México y Estados Unidos para evitar el uso de armas letales, las muertes por parte de agentes estadounidenses son una constante. De acuerdo con la prensa, el principal argumento es el de la defensa propia al ser atacados por migrantes que les tiran piedras.

Concientes de este problema, existen organizaciones humanitarias que ayudan a los migrantes con señalamientos y proporcionan bidones de agua donde las personas puedan abastecerse. Según Humane Borders, una organización que trabaja desde hace varios años en la asistencia de migrantes en la frontera, en el desierto de Sonora, los meses de junio, julio y agosto son los más peligrosos debido a las altas temperaturas. En esos meses se registra el mayor número de muertes; además de las altas temperaturas, ese periodo coincide con un incremento natural del flujo migratorio, debido a que durante el verano la demanda de trabajadores aumenta.

Otra causa importante de muerte en la frontera es por ahogamiento. Varias razones explican que cerca del $15 \%$ de las defunciones se atribuyan a esta causa. En la actualidad no hay muchas opciones, los migrantes tienen que optar entre cruzar por el Río Bravo o aventurarse en el desierto. Y el Río Bravo hace honor a su nombre: como muchos ríos de poco caudal, parece manso en la superficie, pero por abajo está lleno de corrientes y remolinos. Para los migrantes, cruzar «el

2 Ver http://www.gob.mx/wb2/egobierno/egob_grupo_beta 
peligroso río en Texas», como diría Amador de Lira, en un retablo ofrecido a la Virgen de San Juan de los Lagos, es un milagro que hay que agradecer. Por añadidura en la zona de California también se dan muertes por ahogamiento. Como parte del sistema disuasivo los migrantes han sido orientados hacia la zona donde corre el Canal All American (53 Millas) y el Río Nuevo, ambos muy peligrosos. Entre 1995 y 2000 se registraron 177 migrantes muertos por ahogamiento en California (Cornelius 2001).

En la actualidad, la frontera entre México y Estados Unidos se ha convertido en una de las más peligrosas del mundo, ya que en promedio mueren 1.3 personas por día al año. El problema se acentúa debido a la creciente militarización de la frontera, dada la presencia de una mayor cantidad de agentes de la Patrulla Fronteriza, apoyados por seis mil Guardias Nacionales que han sido entrenados para funciones muy distintas a las de vigilar y manejar adecuadamente una frontera. La situación se ha agravado por la presencia de grupos paramilitares, sobre todo en Arizona, que intentan evitar la entrada de indocumentados a territorio estadounidense y que pretenden apoyar las labores de la patrulla fronteriza.

Paradójicamente, los resultados no son nada halagüeños. El balance de varios indicadores, en diferentes periodos, permite concluir, que la solución no se encuentra en el incremento de patrulleros, horas de vigilancia y recursos. De hecho la estrategia implementada ha multiplicado los puntos de vigilancia, ha dispersado los flujos y ha complicado totalmente el panorama. En 1986 con un menor número de patrulleros se realizaron más aprensiones que veinte años después con más del triple de personal.

\section{CUADRO 2}

Indicadores sobre el desempeño de la Patrulla Fronteriza en 1986, 1994, 2005

\begin{tabular}{|c|c|c|c|c|c|}
\hline AÑO & $\begin{array}{c}\text { MIEMBROS } \\
\text { PATRULLA FRONTERIZA }\end{array}$ & $\begin{array}{c}\text { VIGILANCIA } \\
\text { HORAS X MILLONES }\end{array}$ & $\begin{array}{c}\text { APRENSIONES } \\
\text { (EVENTOS) }\end{array}$ & $\begin{array}{c}\text { COYOTES } \\
\text { CAPTURADOS }\end{array}$ & $\begin{array}{c}\text { AGENTES } \\
\text { BOSTAR } \\
\text { RESCATE }\end{array}$ \\
\hline 1986 & 3,693 & 2.4 & 1.671 .458 & 19,274 & \\
\hline 1994 & 4,226 & 3.1 & 1.040 .200 & 14,143 & \\
\hline 2005 & 11,106 & 9.7 & 1.023 .930 & 16,074 & 164 \\
\hline
\end{tabular}

Fuente: Elaborado a partir de información oficial sintetizada por el Mexican Migration Project: http://mmp.opr.princeton.edu/

Este panorama ha preocupado también a las autoridades estadounidenses, que han tratado de paliar el asunto y cambiar su imagen. El principal problema consiste en que es la misma organización la que realiza dos labores contradictorias: por una parte persigue a los migrantes y por otra pretende rescatarlos. En 2005 se habían designado 164 agentes de rescate de BOsTAR (Border Patrol Search Trauma and Rescue) para salvar migrantes en situación de peligro (GAO 2006), lo que es nada comparado con los más de once mil patrulleros que vigilan la fronte- 
ra. Sea como fuere, se ha tomado conciencia del problema y se está avanzando en la contabilidad de las muertes y en algunos programas de salvamento como Border Safety Initiative Tracking System (BSITS); National Center for Health Statistics (NCHS). Esta ambigüedad de algunos organismos también se da en el caso mexicano con la actuación del Grupo Beta en la frontera sur, entre México y Guatemala, donde se supone que personal oficial tiene que proteger, ayudar y custodiar a los inmigrantes indocumentados que transitan por México.

A la esquizofrenia gubernamental hay que sumarle la que se está manifestando en la sociedad. Por una parte existen organizaciones humanitarias que tratan de prevenir las muertes y auxiliar a los migrantes y, por otra, se han multiplicado las organizaciones anti inmigrantes, que incluso quieren hacer justicia con su propia mano. Mientras los voluntarios de Humane Borders, Samaritan Patrol, Paisanos al Rescate y otros, apoyan y rescatan migrantes en peligro; los Minute-Man, American Border Patrol, American Resistance, Send Brick Project y otras tantas, se encargan de denunciar, acosar y perseguir a los migrantes. Sucede algo similar al interior de Estados Unidos, donde algunos condados y ciudades han legislado medidas punitivas contra los indocumentados y en otros casos las autoridades locales protegen a los trabajadores y operan como santuarios (Hopkins, 2007).

La problemática reseñada de la muerte en la frontera está plagada de contradicciones, tensiones y conflictos. En parte porque el tema y la realidad se sitúan en un terreno incierto donde la responsabilidad sobre las muertes y el contenido y extensión de los derechos de los migrantes se presta a interpretaciones contrapuestas, lo que redunda en que nadie asume la responsabilidad por la muerte de personas inocentes. En las fronteras chocan el derecho a libre tránsito, que es un derecho constitucional en la inmensa mayoría de los estados, con el derecho de éstos a controlar y vigilar su territorio. Otro punto de conflicto se da entre la oferta y la demanda de mano de obra, que tradicionalmente se ha entendido como lucha de clases y que en el contexto de la migración laboral, entre naciones, se mueve dentro de los parámetros de una relación bilateral asimétrica.

Otro punto de tensión tiene que ver con la participación de múltiples actores, lo que provoca que las responsabilidades se diluyan. Fácilmente podemos identificar ocho actores diferentes en el que caso nos ocupa: los migrantes, los dos gobiernos involucrados, las dos partes que intervienen en la oferta y la demanda de mano de obra-migrantes y empleadores, las organizaciones que apoyan a los migrantes y las que están en contra y, finalmente, los intermediarios, es decir los coyotes, traficantes o polleros. En esta maraña de instituciones, grupos y personas nadie resulta culpable de nada.

Los traficantes evidentemente se desempeñan al borde de la criminalidad, sin embargo el «crimen» se comete en el momento en que el coyote cruza la frontera. Mientras está en su territorio tiene derecho al libre tránsito, a transportar personas, hospedarlas, orientarlas. Más aún cuando el pago por el servicio se hace una vez que se realiza exitosamente el cruce y no antes. En la mayoría de los casos, se 
trata de un servicio garantizado y requerido por los mismos migrantes. En términos legales estrictos los coyotes cometen el delito cuando cruzan la frontera y no podrían ser perseguidos en su país de origen. En teoría no se puede castigar la intención de cometer un delito. Al parecer el contexto de frontera genera situaciones donde no están claros los límites para el ejercicio del poder y consecuentemente hay una serie de vacíos legales.

Para colmo, ahora también interviene el medio ambiente. Así lo argumenta el gobierno estadounidense al responder al juicio que se promovió en su contra, en la $\mathrm{CIDH}$, donde afirma que las muertes en la frontera se "pueden atribuir a que la gente está mal preparada para cruzar terrenos difíciles», a que los coyotes son responsables por llevarlos por «las peores zonas del desierto", a que «el estado no se puede hacer responsable por las condiciones del terreno donde se realizan actividades ilegales» y que la decisión final descansa en la persona individual que está dispuesta a asumir esos riesgos (CIDH, 2005).

En esta carrera por lograr mejores condiciones de vida para ellos y sus familias suelen sobrevivir los más aptos, los que tienen más recursos, los que están mejor entrenados, los que disponen de mejores guías, en suma, los que tienen «La evidencia», en la gran mayoría de los casos.

Por eso se debe hablar de mortandad en la frontera y no de muerte que suele tener causas que pueden ser explicadas. Cuando se habla de mortandad hay una referencia a la ausencia de causas conocidas, a lo inevitable de la situación, a las condiciones del medio, a una situación que se puede atribuir a la naturaleza y que generalmente se refiere a la especie animal. No obstante, cuando se trata de la mortandad de seres humanos y se alega el principio de «la sobrevivencia del más apto», este fenómeno ha sido caracterizado como «darwinismo social». En nuestros días la selectividad, un tema clásico en la literatura migratoria, parece cobrar un nuevo sentido.

\section{POLIITICAS MIGRATORIAS Y DERECHOS HUMANOS}

Desde el punto de vista de la doctrina de derechos humanos, la muerte de migrantes que intentan llegar a un determinado destino constituye una situación muy preocupante. Más allá del argumento de que no exista manera de atribuir responsabilidad estatal directa en la muerte de estas personas, la situación es moralmente reprensible y por lo tanto debiera ser abordada. Para efectos de este artículo, siguiendo a Jack Donnelly (2003:8) definimos los derechos humanos como aquellos que poseemos como miembros de la especie humana y que permiten la realización/ consecución de una vida digna.

Los estudios que abordan la migración desde la perspectiva de los derechos humanos subrayan que las personas que migran (trabajadores migratorios, refugiados, solicitantes de asilo) se encuentran en una condición de vulnerabilidad estructural (Naciones Unidas Grupo de Trabajo 1998; Ghosh 2003). Esta vulne- 
rabilidad se manifiesta de diversas maneras. Durante su tránsito a los lugares de destino, los migrantes son victimizados por autoridades inescrupulosas o bien por organizaciones delictivas que no sólo lucran de forma ilícita, sino que a menudo maltratan, estafan o despojan a migrantes desesperados que contratan sus servicios. Además, en su afán de burlar la vigilancia de las autoridades, estas organizaciones muchas veces exponen a personas, incluidos mujeres, ancianos y niños, a peligros innecesarios que a veces culminan de forma fatal.

En ocasiones los traficantes abandonan a sus clientes en el desierto o en el mar o los transportan en camionetas o contenedores en condiciones de hacinamiento que a veces les producen muerte por asfixia (Kyle y Dale 2001; CIDH 2002). La victimización se produce sobre todo en áreas fronterizas caracterizadas por altos índices de violencia y poca presencias estatal (Feldmann y Olea 2004). Otro punto que magnifica la vulnerabilidad de los migrantes y que tiene estrecha relación al tema que examinamos es que, la desesperación de estas personas por buscar nuevas oportunidades los lleva a tomar enormes riesgos para llegar a su destino.

En los países de recepción, los migrantes están en una situación de vulnerabilidad ya que muchas veces ignoran las leyes y el idioma y, en ocasiones, deben enfrentar la abierta hostilidad por parte de la población, y/o de las autoridades. Por otro lado, su acceso a derechos económicos y sociales básicos suele ser conculcado en virtud de su nacionalidad y en menor medida por su estatus migratorio (Tarán 2000). La situación es particularmente grave en el caso de las personas indocumentadas, cuya situación legal los expone a mayores abusos.

Para autores como Goodwin-Gill (1989) la vulnerabilidad de los migrantes deriva en parte de su relación con el estado, en tanto éstos establecen arbitrariamente su política migratoria, determinan el conjunto de derechos a los que estas personas tienen acceso de acuerdo a nociones de comunidad y ciudadanía y actúan como protectores y ejecutores de las normas de derechos humanos, a pesar que muchas veces son los propios estados los que vulneran las garantías de estas personas.

\section{TENSIÓN ENTRE PRINCIPIOS FUNDAMENTALES}

En el caso de las muertes de los migrantes en tránsito hacia su destino final, en particular en el caso de las personas que fallecen en la frontera mexicano-estadounidense, nuestro análisis apunta a que, dadas las condiciones especiales en las que perecen estas personas, las normas generales de protección del derecho interno e internacional no son efectivas para garantizar la protección de los derechos de estas personas. Existe un vacío legal relativo a la situación de estas personas tanto en el derecho interno como en el derecho internacional de los derechos humanos. ${ }^{3}$

\footnotetext{
${ }^{3}$ Nuestro argumento deriva en parte de conversaciones que sostuvimos con Juan E. Méndez, ex relator especial de Trabajadores Migratorios y sus Familias de la CIDH.
} 
El vacío legal que caracteriza la situación de los migrantes tiene relación directa con el principio de soberanía. Ésta es el principio rector de las relaciones internacionales modernas desde la firma del Tratado de Westfalia (1648), que puso fin a las sangrientas guerras religiosas que asolaron Europa durante siglos y afirmaron el principio de que la religión de un determinado dominio/estado era impuesta por su regente. La soberanía impone derechos y deberes basados en el concepto de reciprocidad; por tanto, de ella se desprenden derechos como la total independencia en el manejo de los asuntos internos y obligaciones como la no intervención en los asuntos domésticos de otros estados. La soberanía se aplica a un territorio determinado y a la población que habita dicho dominio. En virtud del principio de soberanía, los estados, en tanto formas de organización social moderna, tienen la prerrogativa de decidir sus asuntos internos con total autonomía del mundo exterior (Bull 1977:8-9; Biersteker y Weber 1996 y Krasner 1999). ${ }^{4}$ La única restricción a este principio son deberes de carácter universal que se desprenden de la ratificación de instrumentos internacionales que los estados suscriben voluntariamente en materias tan diversas como derechos humanos, medioambiente, derecho comercial (este importante punto se desarrolla más adelante en esta sección). ${ }^{5}$

Como corolario del principio de soberanía, los estados regulan la entrada y salida de personas desde y hacia sus fronteras. En este sentido, el control del flujo de personas (así como el de información, inversión, comercio) es uno de los factores constitutivos del principio de soberanía. A ese respecto Dauvergne (2004: 592) señala que el control migratorio es uno de los elementos constitutivos de una nación (otros incluyen población, fronteras, y el monopolio del uso legítimo de la violencia). Si bien la delimitación de fronteras y la creación de pasaportes y otros elementos de control migratorio emergieron gradualmente a partir de fines del siglo XVII, la férrea regulación migratoria entre estados geográficamente independientes que conocemos hoy en día se gestó sólo a partir del siglo xx (Dauvergne 2004: 591). Los estados, por ende, tienen la potestad de determinar su política migratoria de acuerdo a sus necesidades y requerimientos. En ese sentido, las políticas migratorias altamente restrictivas existentes en la actualidad en diversos estados se explican por la existencia de una crisis provocada por una sensación de desborde de los flujos migratorios por parte de las autoridades, medios de comunicación y algunos sectores de la población (Weiner 1995: 9-12, Loescher 1993: 7-10; Teitelbaum y Weiner 1995: 16-26).

Sin embargo, la facultad de regular los flujos migratorios en un contexto globalizado e interdependiente se inserta en un proceso social extremadamente comple-

\footnotetext{
${ }^{4}$ Krasner explica que el concepto de soberanía consta de al menos cuatro elementos fundamentales: la organización político/administrativa de un estado y la capacidad de control que ejerce una autoridad dentro de un espacio geográfico determinado (soberanía doméstica); la habilidad de controlar movimientos transfronterizos (especies, mercancías y personas) (soberanía interdependiente); reconocimiento externo (soberanía legal internacional) y la exclusión de actores externos en los asuntos domésticos (soberanía westfaliana) (1999: 9)

${ }^{5}$ El gran jurista estadounidense Louis Henkin se refiere a estas excepciones como una cesión voluntaria de soberanía por parte de los estados (Henkin 1978)
} 
jo donde intervienen diversos actores (estados, personas, organizaciones sociales, empresas, empleadores, sindicatos y diversos grupos de presión), estos actores se caracterizan por poseer intereses múltiples pero a menudo contrapuestos. La naturaleza y características de los flujos migratorios dependen por una parte de los intereses económicos, políticos, sociales y culturales de los estados (receptores, de tránsito y origen) y por otra de los intereses y motivaciones de los propios migrantes (económico, familiar, político) (Weiner 1995; Massey, Durand y Malone 2002; Zolberg 1999).

Si bien la consecución de la política migratoria es una prerrogativa del estado, ésta debe concordar con normas fundamentales de derechos humanos, tanto normas de jus cogens, ${ }^{6}$ como otras derivadas de las obligaciones que voluntariamente contraen los estados al ratificar instrumentos internacionales de derechos humanos. Los estados por ende tienen la obligación de asegurar que el control migratorio y la vigilancia de las fronteras no constituyan en sí mismas una vulneración de los derechos humanos de las personas. Independientemente de la nacionalidad y el estatus migratorio, los estados tienen la obligación de cautelar los derechos fundamentales de los individuos que se encuentran bajo su jurisdicción. Si bien la condición de extranjeros, en particular de personas indocumentadas, implica que no todo el espectro de derechos puede ser exigible (derechos políticos, derechos económicos y sociales), esto no significa que se puedan transgredir derechos fundamentales como el derecho a la vida o a la integridad física (Ghosh 2003, Taran 2000; Méndez, Olea y Feldmann 2006; CIDH 2002). Sassen argumenta que en relación a la fijación de su política migratoria, los estados que cuentan con un estado de derecho están expuestos no sólo a una serie de obligaciones que emanan de normas fundamentales de derechos humanos, sino a la influencia de grupos de presión tanto interno como foráneos (1999).

Diversos instrumentos del derecho internacional de los derechos humanos, tanto universales como regionales, establecen claramente el contenido de las obligaciones de los estados en relación a las personas que migran. Probablemente el más importante sea la Convención Internacional de Protección de los Derechos de los Trabajadores Migratorios y los Miembros de sus Familias (1990), ésta busca ampliar los mecanismos de protección para los trabajadores migratorios y sus familias en diversos temas, a saber: condiciones de explotación laboral y discriminación legal; control de la trata y tráfico de personas y creación de lineamientos claros con relación a los beneficios sociales a los que debieran tener acceso estos individuos. La Convención, sin embargo, tiene bajos niveles de adhesión (sólo 37 estados son parte) y hasta el momento ningún país receptor la ha ratificado.?

${ }^{6}$ Principios de la legislación internacional que ninguna nación puede ignorar, como el genocidio o la trata de esclavos.

7 Convención Internacional de Protección de los Derechos de los Trabajadores Migratorios y los Miembros de sus Familias, Nueva York 18 de Diciembre de 1990. http:/www.ohchr.org/english/ countries/ratification/13.htm 
Existen otra serie de tratados y acuerdos internacionales que prodigan garantías generales a todas las personas, y que se extienden, por ese sólo hecho, a quienes migran. ${ }^{8}$

Más allá de la existencia de estos instrumentos, diversos autores argumentan, persuasivamente, que la legislación internacional existente es insuficiente e inadecuada para cautelar los derechos fundamentales de poblaciones migrantes, ya que existen numerosos vacíos y discriminación en base a la nacionalidad o la pertenencia a una determinada comunidad (en virtud a concepciones religiosas, étnicas) (Hill Maher 2001; Agamben 1998; Ghosh 2003; Taran 2000; Farer 1993). Al respecto Goodwin-Gill sostiene que los migrantes, refugiados y solicitantes de asilo se ubican en la periferia de la protección y que, por lo mismo, sus derechos rara vez son cautelados de forma robusta (1989: 526-528).

Más allá de las obligaciones establecidas en los instrumentos enunciados y de las declaraciones de buena intención de los gobiernos en el sentido de defender los derechos fundamentales de las personas que migran, la contradicción entre los intereses de los estados y de los individuos se ha resuelto hasta ahora claramente en favor de los primeros. Esto deriva de las evidentes asimetrías de poder entre los individuos y los estados, estos últimos amparados en la noción de soberanía, intentan controlar los flujos de entrada a sus territorios (Watson 1992; Krasner 1999).

Al tratar de regular la inmigración, los estados a menudo violan derechos fundamentales de los migrantes. Si bien las arbitrariedades pueden afectar a migrantes en condición regular, los abusos y arbitrariedades se concentran sobre trabajadores indocumentados, refugiados y solicitantes de asilo. Otra serie de violaciones emana de la acción de agentes privados, tanto empleadores como ciudadanos que incurren en actitudes discriminatorias y explotación desmedida sin que el estado, donde estas violaciones ocurren, investigue y sancione a los responsables (Taran 2000; CIDH 2002). En otras oportunidades los estados recurren a sofisticadas estrategias para ocultar dichas violaciones (Feldmann y Olea 2004). Las violaciones a los derechos humanos de inmigrantes muchas veces obedecen al deseo deliberado de los estados de contener y desincentivar la inmigración (Loescher 1993; Farer 1993). Es interesante subrayar que si bien los estados

8 Ver la Declaración Universal de Derechos Humanos de las Naciones Unidas (1948); el Pacto Internacional de Derechos Civiles y Políticos (1966), el Pacto Internacional de Derechos Económicos Sociales y Culturales (1966), la Convención Internacional sobre la Eliminación de Todas las Formas de Discriminación Racial de 1965, entre los más relevantes. Instrumentos regionales como el Convenio Europeo de Derechos Humanos y Libertades Fundamentales, así la Declaración Americana de los Derechos y Deberes del Hombre (1948) y la Convención Americana sobre Derechos Humanos (1978) contienen varias cláusulas generales que protegen de manera genérica los derechos de estas personas. De modo más específico, los Convenios 97 (revisado) de 1949 y 143 de 1975 de la Organización Internacional del Trabajo brindan ciertas garantías de protección a los trabajadores migratorios y sus familias. Sin embargo, estos convenios han sido ratificados por pocos países (41 en el caso del convenio 97 y 18 en el del convenio 143). Asimismo, la Convención de Naciones Unidas en Contra de la Delincuencia Organizada Transnacional, y el Protocolo Contra el Tráfico Ilícito de Migrantes por Tierra, Mar y Aire contienen algunos artículos pertinentes a la protección de personas migrantes víctimas de este tipo de actos ilícitos (CIDH 2002, Bosniak 1990). 
intentan regular la entrada de extranjeros a su territorio, al mismo tiempo abogan por suprimir cualquier tipo de restricción a la salida de personas de sus países de origen (Zolberg 1981 y Dowty 1994).

\section{OPERACIONES DE CONTROL MIGRATORIO Y DERECHOS HUMANOS}

Las muertes de migrantes en la frontera plantean un desafío mayúsculo para quienes intentan brindar protección a estas personas, tanto física como legal, porque la lógica de los abusos deriva en buena parte de la discrecionalidad con la que los estados regulan los procesos migratorios (Ghosh 2003; Méndez, Olea y Feldmann 2006; Goodwin-Gill 1989). En el caso de las muertes en la frontera mexicano-estadounidense, la política de prevención mediante la disuasión ha provocado un desvío de los flujos migratorios hacia áreas despobladas caracterizados por terrenos altamente peligrosos (Spener 2001, 2004, Cornelius 2001; Eschbach et al. 1999. Massey, Durand y Malone 2003). Diversos autores atribuyen la muerte de estas personas a esta política (Smith 1998, Navins 2002, Cornelius 2001, Massey, Durand y Mallone). ${ }^{9}$

Independientemente de si las muertes son el resultado directo o indirecto de una política migratoria (pública), en este caso resulta bastante difícil establecer la responsabilidad estatal dado que estamos en presencia de una colisión de derechos: por un lado, la prerrogativa del Estado de regular los flujos migratorios que ingresan a su territorio $y$, por el otro, el derecho de las personas a migrar, su derecho a la vida, al trabajo y a la integridad personal. El desafío es incluso más significativo porque estamos en presencia de la colisión entre normas fundamentales: el derecho a la vida versus el principio de soberanía. Como resultado de esta tensión, es difícil determinar si la responsabilidad de las muertes es atribuible a los estados (el que desvía los flujos hacia terrenos peligrosos o bien el que expulsa migrantes) o si, por el contrario, son los migrantes los responsables de su predicamento al exponerse a riesgos excesivos.

La CIDH recientemente trató la situación de las víctimas de la frontera mexicano-estadounidense en el caso Víctor Nicolás Sánchez y otros (Operation Gate Keeper) versus Estados Unidos. ${ }^{10} \mathrm{La}$ CIDH analizó una petición a favor del señor Sánchez y otros 354 ciudadanos mexicanos que habrían perecido al intentar cruzar la frontera entre 1995 y $1999 .{ }^{11}$ Si bien la CIDH rechazó la petición en contra de Estados

9 Rodríguez indica que las muertes en la frontera se producían antes de la implementación de la operación Gate Keeper, aunque reconoce que el número era muy inferior.

${ }^{10}$ Caso Víctor Nicolás Sánchez y otros (Operation Gate Keeper) versus Estados Unidos 27 de Octubre de 2005. http://www.cidh.org/annualrep/2005sp/EEUU65.99sp.htm La petición la llevaron a cabo Claudia Smith de la Fundación de Asistencia Rural de California, Paul Hoffman representante del bufete privado Bostwick \& Hoffmann y William Aceves profesor de la Escuela de Derecho de Universidad de California Occidental (California Western School of Law).

${ }^{11}$ En el caso se sólo se acreditaron 240 victimas, la identidad de las 115 restantes no pudo ser establecida. 
Unidos por motivos de procedimiento, ${ }^{12}$ los argumentos de las partes, así como la Opinión Concurrente de su ex presidente, el jurista de Antigua y Barbuda, Clare Roberts, son un interesante punto de partida para el análisis de este problema.

Los peticionarios del caso alegaron que como resultado de la operación Gatekeeper el estado había incurrido en una violación de sus obligaciones internacionales: en concreto, el derecho a la vida, libertad y seguridad personal (CIDH 2005: párrafo 33). Asimismo, sostuvieron que el estado deliberadamente implementó su política migratoria de forma tal que «abusó de su derecho a proteger las fronteras y de su obligación» en materia de derechos humanos provocando la muerte de estas personas (CIDH 2005 párrafo 30). También, sostienen los peticionarios que esta política violó el principio de buena fe en la implementación de las obligaciones internacionales de Estados Unidos, algo que derivó en un abuso de derecho (CIDH 2005 párrafo 32). Finalmente, subrayan, que las obligaciones del estado «existen independientemente de la pretendida culpabilidad de las personas» que intentaban ingresar de manera irregular a territorio estadounidense (CIDH 2005: párrafo 35).

El estado replicó que no había evidencia que su acción hubiese sido deliberada y menos que buscase provocar la muerte de personas para disuadir a potenciales migrantes de ingresar en el futuro a su territorio. Como prueba, Estados Unidos indicó que ha llevado a cabo medidas concretas tendientes a asistir a estas personas, como el establecimiento de brigadas de rescate y primeros auxilios, que buscaban proteger a los migrantes, aún cuando, a su juicio, estas personas cometían un acto ilícito al internarse en su territorio de forma irregular (CIDH 2005: párrafo 40, 41, 42). Por ende, sostuvo Estados Unidos, no podía ser responsabilizado por la decisión individual de ciertas personas de arriesgarse a cruzar la frontera por sectores no habilitados. Estados Unidos alegó que «el derecho a la vida no impone una obligación positiva del estado de evitar de alguna manera todas las pérdidas de vida» y que sus acciones en materia migratoria estuvieron basadas en consideraciones razonables (CIDH 2005: párrafo 43).

Clare Roberts razonó de una manera similar y sostuvo que, si bien los estados deben reconciliar su política migratoria con consideraciones de respeto a normas fundamentales de derechos humanos, históricamente se le reconocía a los estados una considerable discreción en el control de ingreso de los extranjeros a su territorio. En este caso, argumentó Roberts, la responsabilidad por las muertes recaía en la decisión individual de las personas de someterse a los avatares y peligros del viaje. Por ende, sostuvo, no cabía responsabilidad del estado. Roberts manifestó que si bien la situación no implicaba una violación al derecho internacional de los derechos humanos por parte de Estados Unidos, recaía sobre el estado en cuestión -y otros en la región - una obligación moral de aliviar las condiciones que contribuían a la muerte de estas personas (CIDH 2005).

${ }^{12}$ La CIDH declaró la petición inadmisible por la no satisfacción de uno de los requisitos básicos de cualquier petición relativo al agotamiento de los recursos internos (artículo 31 del Estatuto de la $\mathrm{CIDH})$. 
La petición ante la CIDH demuestra de forma prístina nuestro punto, en el sentido que en este tipo de casos el problema se centra en la tensión existente entre principios contrapuestos. Como la muerte deriva de un acto legal, no es fácil probar la responsabilidad estatal en el hecho. Si existe violación, ésta se produce como una externalidad negativa derivada de una acción legal como el control fronterizo que se desprende de uno de los derechos básicos que regula la vida internacional: la soberanía. Por tanto la falta no se encuentra claramente especificada ni en la legislación interna estadounidense ni en el derecho internacional de los derechos humanos.

Ahora bien, reconociendo que estamos en presencia de un vacío legal ya que no existe una norma clara que prohíba específicamente reforzar las fronteras a efectos de controlar los flujos migratorios y, además, que es muy difícil probar la voluntad expresa de las autoridades de desviar los flujos migratorios a efectos de crear un precedente negativo (la muerte de personas) para disuadir a potenciales migrantes de intentar cruzar la frontera, existe un argumento de corte general que podría ser utilizado para determinar la responsabilidad del estado en las muertes. Éste tiene que ver con las obligaciones específicas del estado, no tanto con un presunto acto de omisión.

En su respuesta a los cargos de los peticionarios, es precisamente el estado afectado el que argumenta, no sin razón, que recae sobre él la responsabilidad de hacer todos los esfuerzos razonables (y necesarios) para minimizar amenazas a la vida planteadas por una política de carácter general. Esto se aplica a todas las personas, independientemente de su nacionalidad o estatus migratorio. A ese respecto, Goodwin-Gil argumenta que cuando se trata de derechos humanos fundamentales, la distinción entre nacionales y extranjeros se torna totalmente irrelevante (1989:531).

La Corte Interamericana de Derechos Humanos se ha referido a este punto en concreto, al remarcar la obligación de protección al derecho a la vida a través de medidas de prevención (Medina 2003: 89-99). A este respecto, en el caso Velásquez Rodríguez contra Honduras, ${ }^{13}$ la Corte expresó que el estado tiene la obligación de tomar medidas preventivas específicas destinadas a cautelar el derecho a la vida. Según la Corte, éste deber comprende diversas medidas, desde lo político, administrativo y jurídico a incluso medidas de tipo cultural (educación) que cautelen el derecho a la vida e impongan sanciones para los responsables. En este sentido, para Medina, de este razonamiento se deriva que prevenir se equipara a garantizar este derecho fundamental (Medina 2003: 91). Por otro lado, sostiene Medina, que ya que el estado posee el monopolio legítimo de la fuerza, «las normas que regulan su uso deben ser cuidadosamente elaboradas para prevenir [un] abuso» que

${ }^{13}$ Caso Velásquez Rodríguez, Sentencia de 29 de julio de 1988, Corte I.D.H. (Ser. C) No. 4 (1988). http://www1.umn.edu/humanrts/iachr/C/4-esp.html 
derive en la muerte de un ser humano ya sea de forma deliberada o por negligencia (Medina 2003:92).

El Comité de Derechos Humanos ${ }^{14}$ razonó de forma similar a la Corte Interamericana. En un comentario general sobre el significado y alcance del artículo 6 de la Convención de Derechos Civiles y Políticos relativo al derecho a la vida indica pues este derecho ha sido interpretado, en muchos casos, de forma demasiado estrecha. El «derecho inherente a la vida,» sostuvo el Comité, no puede ser entendido meramente de forma restrictiva, sino que implica acciones positivas por parte del estado para garantizar su pleno goce (Comité de Derechos Humanos 2004: 128).

Siguiendo este razonamiento, en este caso, se puede desprender que la responsabilidad de probar que la política migratoria es razonable y que no lleva a la comisión de una potencial violación de los derechos humanos le corresponde al estado. En otras palabras, la carga de la prueba (burden of proof) se desplaza hacia el estado quien tiene que probar más allá de la duda razonable que sus políticas no constituyen una conculcación de derechos fundamentales. Desde nuestro punto de vista Estados Unidos no pasa la prueba, ya que no ha tomado los recaudos necesarios para evitar las muertes. El hecho de que su política puede no haber sido deliberada no lo exime de responsabilidad, sobre todo si, a la luz de que el número de muertes se ha multiplicado de forma significativa, no ha tomado medidas de fondo para evitar que las muertes continúen. Néstor Rodríguez (2007: 20) plantea de manera muy interesante que existe una responsabilidad moral por parte del agente estatal burocrático, en este caso el Departamento de Seguridad Interior, ${ }^{15}$ al diseñar e implementar una política con resultados no deseados (negativos).

Por último, cabe plantearse también hasta qué punto existe responsabilidad por parte del estado emisor, en este caso México. ¿̇Ha tomado México los recaudos necesarios para evitar que sus nacionales perezcan al intentar cruzar la frontera? Algunos autores plantean la sugerente tesis de que las violaciones de derechos económicos, sociales y culturales en los estados de origen son un factor que impulsa a la gente a migrar (Gzesh 2006). Desde este punto de vista, a México también le cabría una cuota de responsabilidad. ${ }^{16}$ Otra veta relevante tiene que ver con la responsabilidad que le cabe a los estados, tanto de origen como de tránsito y de destino, por combatir las redes de contrabandistas y/o traficantes de personas que ponen en peligro la vida de los migrantes. En este caso, al menos en relación a México, numerosos trabajos muestran que esto no parece ser el caso (Spener 2001; CIDH 2004) y que también existen una serie de contradicciones, interpretaciones, vacíos y anacronismos legales.

${ }^{14}$ Es un cuerpo de 18 destacados juristas que monitorean el cumplimiento de la Convención de Derechos Civiles y Políticos (Donnelly 2003: 133).

${ }^{15}$ Entre 1993-2003 le responsabilidad recayó en el Servicio de Inmigración y Naturalización.

${ }^{16}$ Sobre el argumento que México incurre en una política similar en su frontera sur en el sentido de dejar el control en manos de agentes particulares que victimizan a los migrantes y por ende inhiben la migración ver Feldmann y Olea 2004. 
La muerte de migrantes que intentan llegar a un destino que les ofrezca condiciones de vida dignas constituye una situación inaceptable. Cada año mueren cientos de personas en diversos puntos del orbe, de los que mueren ahogados en los océanos casi no hay información, pero sí se pueden rastrear y contabilizar los casos de los migrantes que mueren al exponerse a terrenos inhóspitos, a climas extremos y los que son abandonados o asesinados por maleantes y traficantes. La desesperación por escapar de condiciones miserables en sus comunidades de origen lleva a estas personas a tomar riesgos enormes, en su afán por burlar la vigilancia de las fronteras por parte de los agentes estatales. Más allá de la evidente resistencia física y psicológica y el elevado umbral de sufrimiento que muestran estas personas, los riesgos asociados al periplo migratorio magnifican la condición de vulnerabilidad estructural que padecen estas personas. En estas condiciones, sólo los más fuertes sobreviven.

Las vicisitudes a las que se exponen las personas que intentan cruzar la frontera tienen como resultado la lesión de derechos fundamentales como el derecho a la vida y a la integridad física. Las protecciones para esta población son endebles porque existe un inadecuado reconocimiento legal de los derechos humanos de los migrantes. La legislación internacional vigente es insuficiente y presenta numerosos vacíos que impiden a los migrantes el pleno goce de sus derechos fundamentales. De forma más general, no existe un régimen internacional que regule de forma humana e idónea los ingentes flujos migratorios

Se ha argumentado que en el caso de la muerte de personas que intentan ingresar, de manera no autorizada, a un determinado territorio se manifiesta la colisión normas fundamentales: por un lado el derecho a la vida, integridad física, trabajo y libertad de movimiento de los migrantes y por el otro, el derecho de los estados a regular los flujos migratorios que emana del principio de soberanía. La sociedad internacional no ha sido capaz de resolver esta contradicción a modo de avanzar en una institucionalidad que lleve a la consecución de ambos principios de forma armónica, vale decir, respetar la prerrogativa de los estados de regular los flujos migratorios, pero cautelando los derechos fundamentales de las miles de personas que migran.

La ausencia de una solución a este problema se expresa en la inexistencia de un marco legal, tanto en el derecho interno como en el derecho internacional de los derechos humanos, que proteja la vida y la integridad de las personas que migran. No existe una norma específica que limite la forma en la que los estados regulan la entrada de personas a su territorio y custodian sus fronteras. Sólo existe de forma general una obligación de realizar todos los esfuerzos razonables (y necesarios) para reducir posibles amenazas a la vida, planteadas por una política de carácter general. El problema se agrava porque en la actualidad hay un repunte en los flujos migratorios irregulares a nivel mundial especialmente en los que provienen de los países 
pobres del sur y se dirigen a las potencias económicas e industriales del norte. De manera paralela se han intensificado los controles estatales y las políticas migratorias restrictivas. Las economías de los países centrales demandan trabajadores, mano de obra barata, y proveen de empleo a los migrantes; pero los estados no reconocen los derechos humanos y laborales de los migrantes. En este contexto se requiere de una revisión profunda de la legislación internacional que subsane los numerosos vacíos legales que impiden a los migrantes el pleno goce de sus derechos fundamentales.

Si bien no existe una legislación adecuada, es primordial impulsar de manera rápida la solución de este problema, por razones de carácter ético. En parte se debería avanzar en prácticas concretas de los estados que pongan fin a esta lamentable situación. Esto requerirá de un enorme liderazgo y de visión por parte de las autoridades de diversos estados, sobre todo receptores, que son vulnerables a grupos de presión que demonizan a los migrantes.

Eventualmente, con imaginación y voluntad política se podría crear un marco que le dé garantías a los estados, pero que evite que la cadena de muertes se siga propagando, no sólo en la frontera mexicano-estadounidense sino en otros puntos críticos.

\section{BIBLIOGRAFÍA}

Agamben, Giorgio, (1998), Homo Sacer: Sovereign Power and Bare Life, Stanford, Stanford University Press.

Biersteker, Thomas y Cynthia Weber (1996), «The Social Construction of State Sovereignty», en State Sovereignty as a Social Construct, Thomas Biersteker y Cynthia Weber (eds.), pp. 1-22, Cambridge, Cambridge University Press.

Bosniak, Linda S. (1990), «Human Rights, State Sovereignty and the Protection of Undocumented Migrants Under the International Migrant Workers Convention», International Migration Review, 25 (4): 737-771.

Bull, Hedley (1977), The Anarchical Society, Nueva York, University of Columbia Press. Castles, Stephen y Mark Miller (2003), The Age of Migration (3a. ed.), Nueva York, The Guilford Press.

Comisión Interamericana de Derechos Humanos (CidH) (2005), Caso Víctor Nicolás Sánchez y otros (Operation Gate Keeper) versus Estados Unidos 27 de Octubre de 2005, http://www.cidh.org/annualrep/2005sp/EEUU65.99sp.htm

(2004), Relatoría Especial de Trabajadores y Miembros de sus Familias, Visita a Países, México, http://www.cidh.org/Migrantes/2003.sp.cap.5c.htm

(2002), Relatoría Especial de Trabajadores y Miembros de sus Familias, Estudios Temáticos/Investigaciones. Migración y Derechos Humanos. http://www.cidh.org/ Migrantes/migrantes.00sp.htm\#MIGRACIÓN

Comité de Derechos Humanos (2004), Compilation of General Comments and General Recommendations Adopted by Human Rights Treaty Bodies. HRI/GEN/1/Rev.7 12 May 2004 General Comment No. 6: Article 6 (Right to life). 
http://www.unhchr.ch/tbs/doc.nsf/(Symbol)/ca12c3a4ea8d6c53c1256d500056e56f ?Opendocument

Cornelius, Wayne (2001), "Death at the Border: Efficacy and Unintended Consequences of US Immigration Control Policy», Population and Development Review, 27 (4):661-685.

(1994), Controlling Immigration. A Global Perspective, Stanford, Stanford University Press.

Cortés, Mario (2003) «Política inmigratoria de México y Estados Unidos y algunas de sus consecuencias», en Región y Sociedad, vol. xv, núm. 27.

Dauvergne, Catherine (2004), "Sovereignty, Migration and the Rule of Law in Global Times», Modern Law Review, 67 (4): 588-615.

Donnelly, Jack (2003), Universal Human Rights in Theory and Practice, Ithaca, Cornell University Press.

DowTy, Alan (1994), Closed Borders: The Contemporary Assault on Freedom of Movement, New Haven, Yale University Press.

Durand, Jorge y Douglas S. Massey (2003), Clandestinos. Migración México Estados Unidos en los albores del siglo XXI, México D.F., Editorial Miguel Ángel Porrua, Universidad de Zacatecas.

El UniverSal (2007), Son migrantes mexicanos cuerpos hallados en incendios, 27 de Octubre http://www.el-universal.com.mx/notas/457696.html

Eschbach, Karl, Jacqueline Hagan, Nestor Rodriguez y Rubén Hernández-León (1999), "Dead at the Border», en International Migration Review, 33(2) 430-454.

et al. (2001), "Causes and Trends in Migrant Deaths along the U:S.-Mexican Border, 1985-1998», Working Paper No. WP01-4, Center for immigration Research University of Houston, Houston, Tex.

FARER, Tom (1993), «How the International Systems Copes with Involuntary Migration: Norms, Institutions and State Practice», en Threatened People's, Threatened Borders: World Migration and US Policy, Michael Teitelbaum y Myron Weiner (eds.), 257-292. Nueva York, W.W. Norton \& Company.

Feldmann, Andreas y Helena Olea (2004), «New Formulas, Old Sins: Human Rights Abuses Against Migrant Workers, Asylum Seekers, and Refugees in the Americas», en Human Rights From the Margins: Critical Interventions, Neve Gordon ed., pp. 129-59. Lanham, MD., Lexington Books.

(gao) United States Government Accountability Office (2006), "lllegal Immigration. Border-Crossing Deaths Have Doubled Since 1995; Border Patrol's Efforts to Prevent Deaths Have not Been Fully Evaluated», Report to the Honorable Bill First, Majority Leader, U.S. Senate.

Goodwin-GiLl, Guy (1989), «International Law and Human Rights: Trends Concerning International Migrants and Refugees», International Migration Review 23 (3): 526-546.

Grosh, Bimal (2007), Human Rights and Migration: The Missing Link, Conferencia Dictada en la Universidad de Chicago, 12 de octubre.

GHosh, Bimal (2003), Elusive Protection, Uncertain Land: Migrants' Access to Human Rights. Geneva, International Organization for Migration.

Gzesh, Susan (2006), «Transforming Migration Policy: A Holistic Approach Using Human Rights», Segundo Coloquio Sobre Migración y Desarrollo, Morelos, 26 de octubre. 
Henkin, Louis (1978), The Rights of Man Today, Boulder, Westview Press.

Hill-MaHer, Kristen (2001) «Who has a Right to Rights? Citizenship's exclusions in an age of migration», en Globalization and Human Rights, ed. Alison Brysk, pp. 19-43. Berkeley, University of California Press.

Hopkins, Daniel J. (2007), "Threatening changes: Explaining where and when Immigrants Provoke Local Opposition", Unpublished paper. Presented at the 2007 annual conference of the Midwest Political Science Association and the American Political Science Association.

Krasner, Stephen (1999), Sovereignty: Organized Hypocrisy, Princeton, Princeton University Press.

Kyle, David y John Dale (2001), «Smuggling the State Back: Agents of Human Smuggling Reconsidered», en Global Human Smuggling: Comparative Perspectives, David Kyle y Rey Kislowski (eds.), Baltimore, Johns Hopkins University Press.

Loescher, Gil (1993), Beyond Charity: International Cooperation and the International Refugee Crisis, Oxford, Oxford University Press.

Marroni, María da Gloria y Guillermo Alonso Meneses (2006) «El fin del sueño americano. Mujeres migrantes muertas en la frontera México Estados Unidos» en Migraciones Internacionales, vol. 3, núm. 3, enero-julio, pp. 5-30.

Massey, Douglas, Jorge Durand y Nolan Malone (2002), Beyond Smoke and Mirrors, Nueva York, Russell Sage Foundation.

Medina, Cecilia (2003), La Convención Americana, Teoría y Jurisprudencia: Vida, Integridad Personal, Libertad Personal, Debido Proceso y Recurso Judicial, Santiago de Chile, Centro de Derechos Humanos Universidad de Chile.

Méndez, Juan R., Helena Olea y Andreas Feldmann (2006), «International Standards of Due Process for Migrant Workers, Asylum Seekers, and Refugees», en Human Rights, Refugees and Displaced Persons in Process: Essays in Honor of Joan Fitzpatrick and Arthur, Helton, Anne Bayefsky (ed.), pp. 459-476, Leiden, Brill Academic Publishers.

Mexican Migration Project mmp (2007), http://mmp.opr.princeton.edu/ 114 comunidades NaCiones Unidas. Grupo de Trabajo Intergubernamental de Expertos en los Derechos Humanos de los Migrantes, Informe E/CN.4/AC.46/1998/5, párrafo 28; Naciones Unidas, Derechos Humanos de los Migrantes, Informe E/CN.4/2000/82, párrafo 13. Navins, Joseph (2002), Operation Gatekeeper: The Rise of the 'Illegal Alien' and the Making of the US-Mexico Boundary, Nueva York, Routledge.

Newland, Kathleen (2003), Troubled Waters: Rescue of Asylum Seekers and Refugees at Sea, Migration Policy Institute http://www.migrationinformation.org/Feature/ display.cfm? $\mathrm{ID}=80$

ReYES, Belinda et al. (2002), "Holding the line» The Effect of the recent Border Built-up on Unauthorized Immigration» Public Policy Institute of California, San Francisco, Calif., 2002.68

Rodriguez, Nestor (2007), Border Issues and Human Rights: Issues of an Enforcement Strategy, Unpublished Paper, Center for Immigration Research, University of Houston. Ryo, Emily (2006) Through the Back Door: Applying Theories of Legal Compliance to Illegal Immigration During the Chinese Exclusion Era, 31 Law \& Soc. Inquiry 109 (Law and Social Inquiry Winter, 2006). 
Sanjeeb Sapkota et al. (2006), «Unauthorized Border Crossing and Migrants Deads: Arizona, New México, El Paso, Tex., 2002-2003» American Journal of Public Health, July 2006.

SASSEn, Saskia (1999), «Beyond Sovereignty: De Facto Transnationalism in Immigration Policy», European Journal of Migration and Law, 1: 177-198.

Scharf, Daniel A. (2006), Case for human borders: two decades of death and illegal activity in the Sonoran Desert For hUMANe BORDERS: TWO DECADES OF DEATH AND ILLEGAL. Western Reserve Journal of International Law, 2006, vol. 38, Issue 1, p. 141-172, 32 pp.; (AN 20903839).

Sмrth, Claudia E. (1998), Operation Gatekeeper and Migrants Death», California Association of Human Relations Organizations Newsletter http://www.cahro.org/html/decjan98-3.html

Spener, David (2001), «Smuggling Migrants Through South Texas: Challenges Posed by Operation Rio Grande», en Global Human Smuggling: Comparative Perspectives, David Kyle y Rey Koslowski (ed), Baltimore, Johns Hopkins University Press.

(2004), «Mexican Migrant-Smuggling: A Cross-Border Cottage Industry», 2004, Journal of International Migration and Integration, 5:3: 295-320.

TARAn, Patrick (2000), «Human Rights of Migrants: Challenges of a New Decade», International Migration 38 (6): 7-51.

Teitelbaum, Michael (1984), Immigration Refugees and Foreign Policy', International Organization 38 (3), pp., 429:450.

y Myron Weiner (1995), «Introduction», en Threatened People's, Threatened Borders: World Migration and US Policy, Michael Teitelbaum y Myron Weiner (eds.), pp. 13-38, New York, W.W. Norton \& Company.

Watson, Adam (1992), The Evolution of International Society, London, Routledge.

Weiner, Myron (1995), The Global Migration Crisis: Challenges to States and Human Rights, Nueva York, Harper Collins College Publishers.

(1996), «Ethics, National Sovereignty and the Control of Immigration», International Migration Review (30) 1, pp. 171.

Zolberg, Aristide (1999), "The Politics of Immigration Policy,» American Behavioral Scientist, 42 (9): 276-280.

(1981), «International Migrations in Political Perspective», en Global Trends in Migration: Theory and Research on International Population Movements, Mary M. Kritz, Charles B. Keely y Silvan M. Tomasi (eds.), S, pp. 3-27, Nueva York, The Center for Migration Studies. 
